\title{
Polarization Diversity on ESM Systems
}

\author{
Rafael Gonçalves Licursi de Mello \\ Força Aérea Brasileira, Laboratório de RF-UFSC rafael.licursi@posgrad.ufsc.br \\ Cynthia Junqueira \\ P\&D Department - Espectro Ltda., FEEC-UNICAMP cyjunqueira@espectro-eng.com.br
}

\begin{abstract}
Polarization diversity antenna arrays are applied to electronic support measures (ESM) systems since the 1980s. However, even today modern systems are conceived with the employment of the traditional and inconvenient spiral antennas and there are no studies that evidences most of the benefits of this technique applied to them. This paper aims at showing not only the advantages on polarization matching issues, but also the benefits in gain, in simpler truncation effect problems and in costs and time spent on design and production. After a brief approach strictly applied to ESM of the main features of spiral antennas and bow-tie antennas compounding a polarization diversity array, equations of the power available from the last were developed from the concept of effective length. The results demonstrate that the array could provide a power $8.7 \mathrm{~dB}$ higher than a modern spiral would to an ESM system when it receives vertical or horizontal linearly polarized waves, which multiplies by 2.7 the range of the system, besides also presenting a better performance in case of circularly polarized incident waves. This work has contributed to propose a replacement to the very common use of spiral antennas on the upcoming ESM system projects. This study is recommended for the areas of Electronic Warfare, Electromagnetic Devices and Applications and Ultra-Wideband Antennas.
\end{abstract}

Index Terms - bow-tie antennas, electronic support measures, polarization diversity antenna array, spiral antennas.

\section{INTRODUCTION}

Even though one of the Electronic Warfare trends is to explore frequency ranges beyond radiofrequency $(\mathrm{RF})$, the eternal conflict between radars and electronic support measures (ESM) systems, which began on the $20^{\text {th }}$ century, still has a prime importance in the military context. On this conflict, every wasted $\mathrm{dB}$ could mean an unsuccessful detection; every non-well calibrated radiation pattern anomaly could succeed in an inaccuracy of measurement. These can result in a mission failure.

Polarization diversity antenna arrays are applied to ESM systems since the early 1980s. However, although exist lots of studies about this technique, most of them are about mobile phone networks [1], RFID [2] and WLAN [3] applications, HF [4] and optical [5] communications. None of them studies most of the benefits of polarization diversity applied to ESM systems. Even those focused on ultrawideband antennas [6], that would be interesting to these military systems, do not. Besides that, many modern ESM systems are still designed with the traditional and inconvenient spiral antennas.

This brief shows not only the mathematical demonstration of combining signals generated in a couple of bow-tie antennas, but also the benefits in gain, in less unfavorable truncation effect issues, 
and in the fact that they are cheaper and easier to be designed, computer simulated and produced, compared to the classical spirals.

The goal of this effort, besides demonstrating the above subjects, is to explicit that the ultimate technologies allow this traditional and inconvenient employment to be replaced by polarization diversity antenna arrays.

\section{ANTENNAS APPLIED TO ESM SYSTEMS}

\section{A. Electronic Support Measures Systems}

The ESM systems are designed to intercept, characterize, identify and locate RF emitters through the passive monitoring of the electromagnetic environment. They could be considered successful when confronting the most advanced modern radars as they "detect while staying undetected", and, to do that, they must be able to receive and process the weakest and furthest transmitted signal [7].

The performance of such equipment depends, among other factors, on the sensitivity and the internal losses inherent to the components of their receivers, but, specifically, the antennas and their polarization matching with the incident waves are the first aspects to be considered [8].

Fig. 1 shows a generic ESM system architecture. On this paper, the receiver, the processor, the database, the special function generator and the displays are not under analysis.

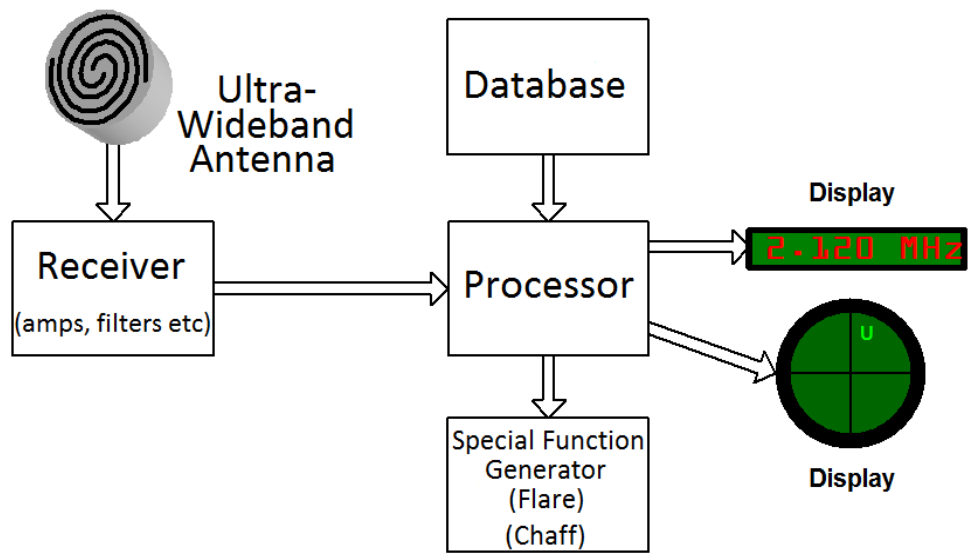

Fig. 1. Generic ESM system.

Depending on the application, an ESM system can measure various parameters of received signals. One of them has tactical and strategical importance and it is known as direction of arrival (DOA). It reveals the direction from where the radar emits the signal.

The simplest reliable way of getting DOA is called the amplitude comparison method, in which it is necessary that the system has an antenna array covering the sector of interest. The antennas must have a characteristic radiation pattern, with a main lobe basically covering its respective sector of interest, and less expressive side lobes [9]. Fig. 2, obtained on CST software [10], illustrates a typical radiation pattern of antennas that would be appropriate to compound an array of six units, each one responsible for covering $60^{\circ}$ of azimuth. The beamwidth of $85.4^{\circ}$ covers the sector of interest. The main lobe radiation is $9.58 \mathrm{~dB}$ more efficient than the side one. 


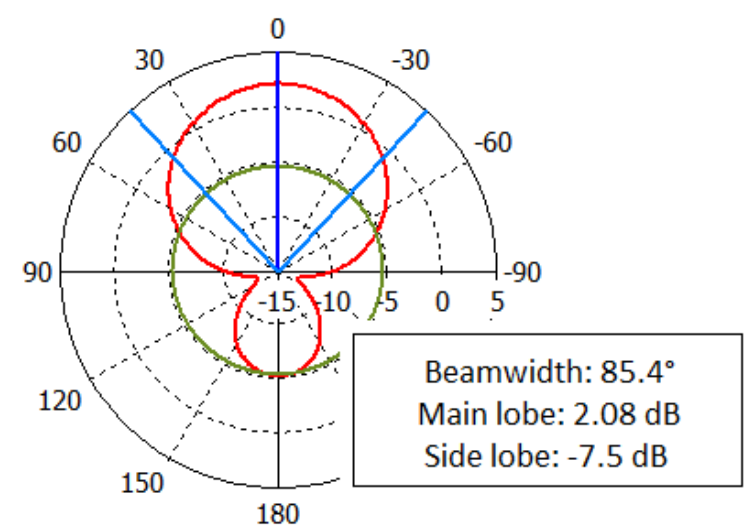

Fig. 2. Typical radiation pattern of an appropriated antenna to the amplitude comparison method.

Fig. 3 illustrates the amplitude comparison method. When the six-unit array, represented in the left, receives a $\vec{V}_{i}$ signal, a specific voltage is generated in each antenna, in reason of their angular disposition. The evaluation of DOA is made by the ESM system as shown in the right.

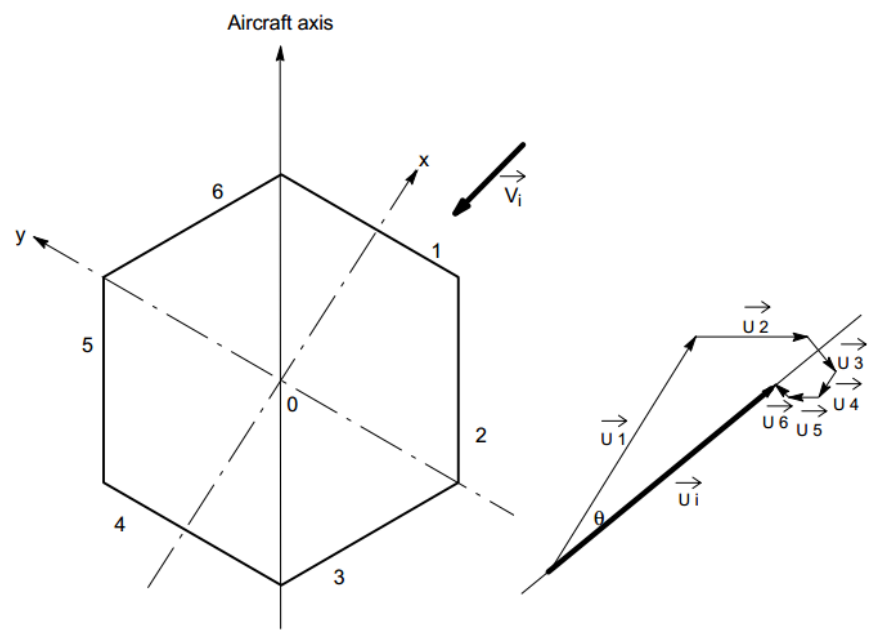

Fig. 3. The amplitude comparison method.

The calibration of the system and the measurement of DOA through this method will be as easy and accurate as the radiation patterns of each array unit are regular ones. If the patterns do not reach a minimum regularity, this important parameter will be unmeasurable.

The ESM systems may be installed on several types of platform, such as warships, trucks, aircraft and satellites. Some, especially the last two, could present serious limitations in weight and volume.

\section{B. Ultra-Wideband Antennas}

The ESM systems are made to cover a wide range of frequencies and thus confront all the radars on that whole range. To achieve this, ultra-wideband antennas, which operate in the proportion of 20:1, or even $40: 1$, are usually employed.

These objects were developed from theoretical antennas, called by Rumsey as frequency independent antennas [11]. To be frequency independent, antennas must be determined by angles, must behave as if they had infinite dimensions and must have less periodic variation as possible. 
As it is not possible the real manufacturing of an infinite-dimensional antenna, as it is limited to a practical size, the truncation effect arises. It comes from the unwanted reflection of the electrical current at the end of the antenna conductors, causing several drawbacks, depending on the geometry of the radiation antenna elements.

\section{Measurement of DOA Using Spiral Antennas}

The most common type of antenna used on ESM systems is the spiral antenna backed by a conducting plane reflector, as illustrated in Fig. 4. They are employed because of their capability of receiving waves with different kinds of polarization and their ultra-wideband operation, thus covering a large class of radars, besides their low weight and volume.

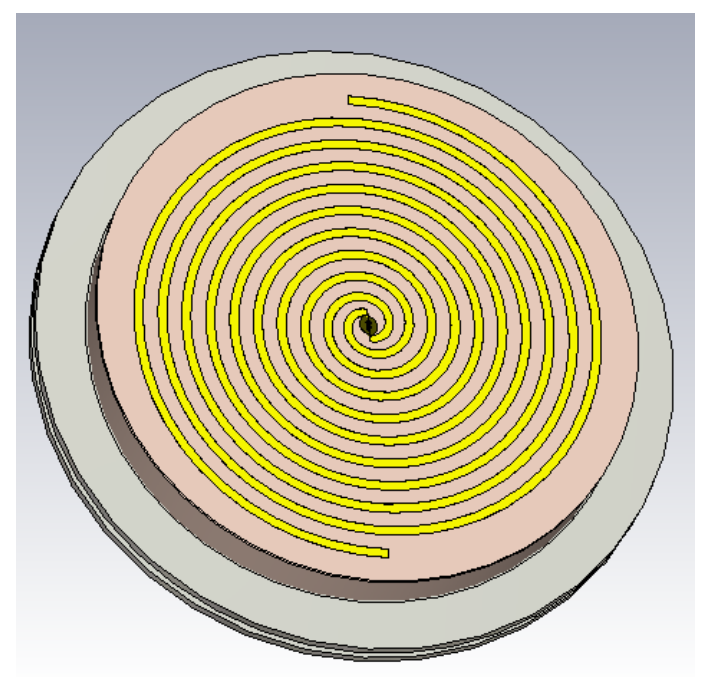

Fig. 4. Spiral antenna backed by a conducting plane [10].

The typical radiation pattern of such antennas is similar to that of Fig. 2. Modern variations of these devices could achieve a gain around $6.8 \mathrm{dBi}[12]$.

The radiation elements geometry provides it right or left-handed circular polarization (RHCP or LHCP), according to the rotation of the spirals. Because of that, this type of antenna causes attenuation of $3 \mathrm{~dB}$ (if its axial ratio is equal to $0 \mathrm{~dB}$ ) in case of linearly polarized (LP) incident waves and it is practically unable to receive unmatched circularly polarized waves [13].

The calculation of DOA on systems which use spiral antennas is hampered when truncation effect is significant. The current reflected at the open-ends runs through the conductive spirals in reverse, producing new radiation polarized in the opposite hand of the original. This new radiation interacts with the first, which induces irregularities in the radiation pattern, moves the maximum radiation axis and increases the axial ratio. To reduce the truncation effect is one of the greatest challenges of designers.

The lowest operating frequencies of a spiral, related to incident signals with the largest wavelengths, are defined by its external diameter. The highest frequencies, related to the shortest wavelengths, are determined by the precision of the conductors in the center of the spiral. This turns 
the manufacturing process also a major challenge, since it must be as accurate as it is expected to achieve the highest frequencies.

Currently, spiral antennas can be developed, optimized and analyzed with the aid of electromagnetic analysis software [14], which reduces costs and time spent on the project, besides increasing its quality. Nevertheless, the dimensions of the conductors arranged in the shape of spirals together with the complexity and the precision of techniques to reduce truncation effect contribute for these simulations to be a slow process and to require high performance computers. These factors also complicate the manufacturing and increase time and costs spent.

\section{Measurement of DOA Using Polarization Diversity Bow-Tie Antenna Arrays}

Bow-tie antennas could be applied in couples, orthogonally to each other, in order to satisfy the need of ultra-wideband operation of ESM systems. These antennas are simplifications of biconical antennas flattened in a plane, and they could also be compared to dipoles defined by angles, which meet the conditions detailed by Rumsey. Fig. 5 exemplifies a bow-tie.

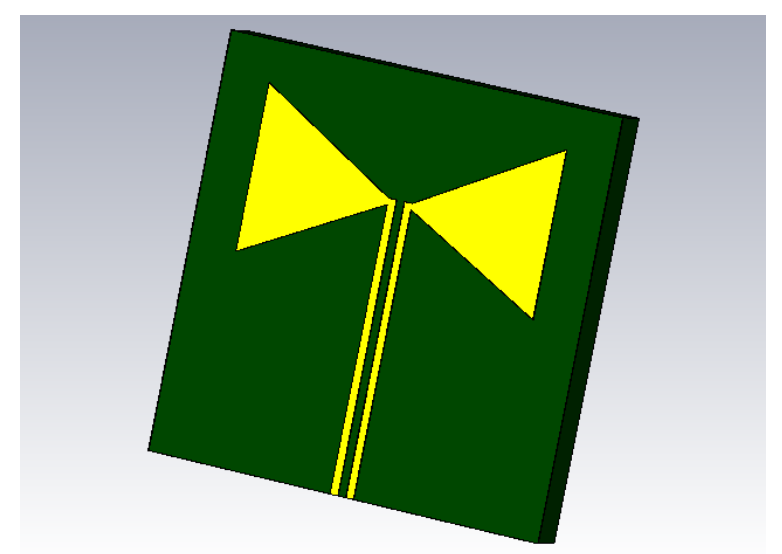

Fig. 5. Bow-tie dipole antenna.

The typical radiation pattern of a bow-tie is similar to a dipole one, i.e., omnidirectional. Reflectors may be used in order to mold it and make it suitable to obtain DOA through the amplitude comparison method. Modern devices have gain around $9.5 \mathrm{dBi}[15]$.

These antennas are LP. Therefore, they receive RHCP and LHCP waves with attenuation of $3 \mathrm{~dB}$, and they are practically unable to receive LP waves in the transverse direction [13]. These characteristics make one single isolated antenna not proper to constitute ESM systems, because it would be impaired to detect a number of radars. However, by combining orthogonally two of them this issue ceases. Advances on the area of miniaturization of antennas [16] and RF components [17] have made possible previously inconceivable space arrangements. Because of that, the weight and volume restrictions from aircraft and satellites nowadays may be circumvented.

The two signals generated by the orthogonally arranged antennas must pass through a diversity combiner switch. On this paper, the switch response is in equal-gain without a phase delay between the two input signals or with it. Fig. 6 and Fig. 7 illustrate these two ESM system architectures. 


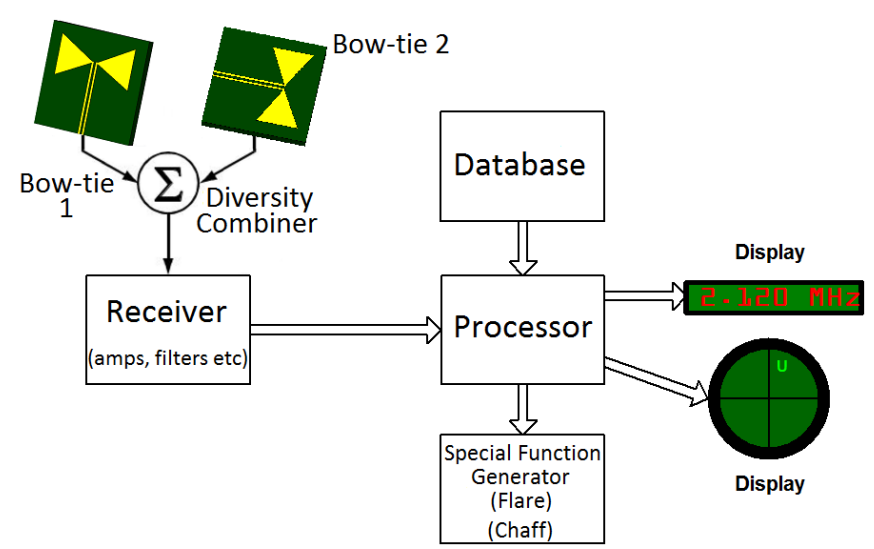

Fig. 6. Polarization diversity bow-tie antenna array on a generic ESM system, without phase shifting.

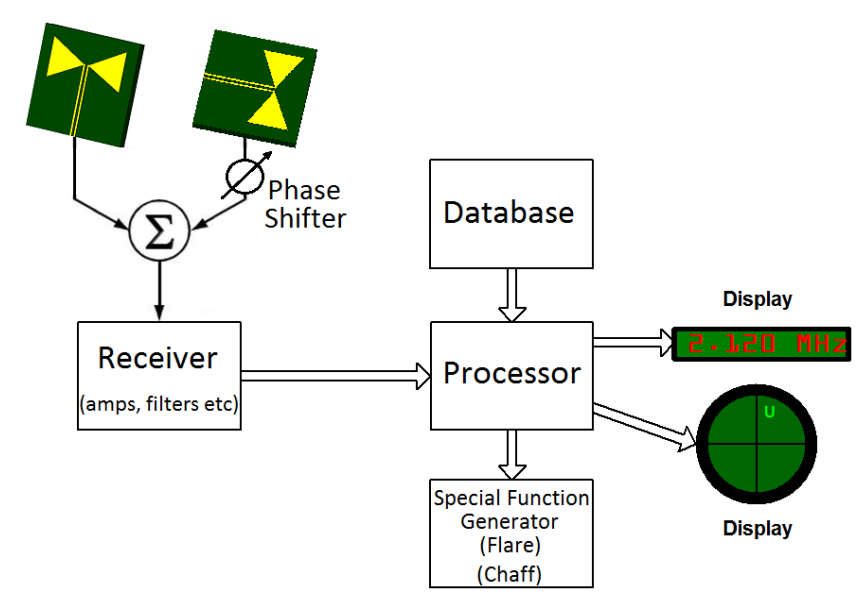

Fig. 7. Polarization diversity bow-tie antenna array on a generic ESM system, with phase shifting.

Truncation effect decreases the gain of bow-ties, as seen in the spirals. However, the axial ratio problem does not appear on the polarization diversity antenna array because each antenna is responsible for receiving one of the components of the incident signal.

The simplicity of the radiator elements of the bow-ties geometry, unlike the spirals, does not require complicated manufacturing processes. It also facilitates the computer simulation of these objects. Because of that, their design and manufacturing process tend to spend less time and costs than that of the spirals.

\section{Mathematical Demonstration of Polarization Matching of Combiner Switch}

\section{A. Enunciation}

Consider $z=0$ the position located in the open-ends of a couple of antennas, both LP, one of them disposed vertically $V$ and the other horizontally $H$. Consider that they feed a diversity combiner switch, which can operate in one of two forms mentioned above, i.e., providing on its output the sum voltage of the two input signals without phase shifting, or providing this sum with phase delay of $90^{\circ}$ in one of them. For this particular example, the $V$ antenna input signal is delayed. 
For comparative purposes, consider signals with different types of polarization but with the same intensity that could stimulate the open-ends of such antennas. On position $z=0$, the electric field $\vec{E}$ as a function of time $t$ of a LHCP incident wave, of a RHCP incident wave, of a linearly polarized wave in a plane inclined at $45^{\circ}$ between the orthogonal antennas (ILP), of a wave with horizontal linear polarization (HLP) and other with vertical linear polarization (VLP) could be respectively represented and defined as in (1) to (5) below:

$$
\begin{gathered}
\vec{E}_{L C P}(t)=E_{0} \cos (\omega t) \hat{x}+E_{0} \cos \left(\omega t-\frac{\pi}{2}\right) \hat{y} \\
\vec{E}_{R C P}(t)=E_{0} \cos (\omega t) \hat{x}+E_{0} \cos \left(\omega t+\frac{\pi}{2}\right) \hat{y} \\
\vec{E}_{I L P}(t)=E_{0} \cos (\omega t) \hat{x}+E_{0} \cos (\omega t) \hat{y} \\
\vec{E}_{H L P}(t)=\sqrt{2} E_{0} \cos (\omega t) \hat{x} \\
\vec{E}_{V L P}(t)=\sqrt{2} E_{0} \cos (\omega t) \hat{y}
\end{gathered}
$$

where $E_{0}$ is the electric field amplitude and $\omega$ is the angular frequency. The $\sqrt{2}$ factor applied to HLP and VLP waves aims to consider, for comparative calculation purposes, the same amplitude and the same incident power for all types of the above mentioned waves.

The voltage generated on the open-ends of an antenna can be evaluated from the concept of effective length $l$, defined as how much voltage $V_{g}$ it would generate when excited by a wave with electric field amplitude $E$ [15]. Thus:

$$
V_{g}=l E
$$

Applying the incident waves component of unit vector $\hat{x}$ on the horizontally disposed antenna, $H$, and the component of unit vector $\hat{y}$ on the vertically disposed antenna, $V$, both of effective length $l=l_{0}$, the voltage phasor generated in $z=0$ specified by acronyms for different occasions are:

$$
\begin{gathered}
V_{g H}(L C P)=l_{0} E_{0} \\
V_{g V}(L C P)=-j l_{0} E_{0} \\
V_{g H}(R C P)=l_{0} E_{0} \\
V_{g V}(R C P)=j l_{0} E_{0} \\
V_{g H}(I L P)=l_{0} E_{0} \\
V_{g V}(I L P)=l_{0} E_{0} \\
V_{g H}(H L P)=\sqrt{2} l_{0} E_{0} \\
V_{g V}(H L P)=0 \\
V_{g H}(V L P)=0 \\
V_{g V}(V L P)=\sqrt{2} l_{0} E_{0}
\end{gathered}
$$


For matched impedances $Z_{0}$, the voltage drop across the internal impedance of the generator - the antennas in this case - consists of $50 \%$ of the total. Thus, only $50 \%$ of voltage will reach the proposed switch, whose $V_{\text {out }}$ equal-gain diversity combining equation when there is no phase shifting, like that of Fig. 6, is presented in (17):

$$
V_{\text {out }}=a\left[\frac{V_{g H}}{2}+\frac{V_{g V}}{2}\right]
$$

where $a$ is the conversion factor of the combiner switch. For input signals combined with phase delaying on $V$, like that of Fig. 7, the equation is:

$$
V_{\text {out }}=a\left[\frac{V_{g H}}{2}+(-j) \frac{V_{g V}}{2}\right]
$$

The instantaneous electrical power $P_{\text {out }}$ available at the output of the switch can be depicted as:

$$
P_{\text {out }}=\frac{\left[\operatorname{Re}\left(V_{\text {out }} e^{j \omega t}\right)\right]^{2}}{Z_{0}}
$$

\section{B. Numerical Calculation}

Consider $E_{0}=1 \mathrm{~V} / \mathrm{m}, \omega=10^{9} \mathrm{rad} / \mathrm{s}, l_{0}=1 \mathrm{~m}, a=1 / \sqrt{2}$ and $Z_{0}=50 \Omega$. Disregard the mutual coupling, related on literature [6] to be less than $-21 \mathrm{~dB}$ in a modern polarization diversity array of antennas with similar radiation pattern and ultra-wideband behavior of bow-ties.

With the aid of Matlab software [18], numerically calculating the developed equations, the graphics shown in Fig. 8 from (a) to (e) are presented.

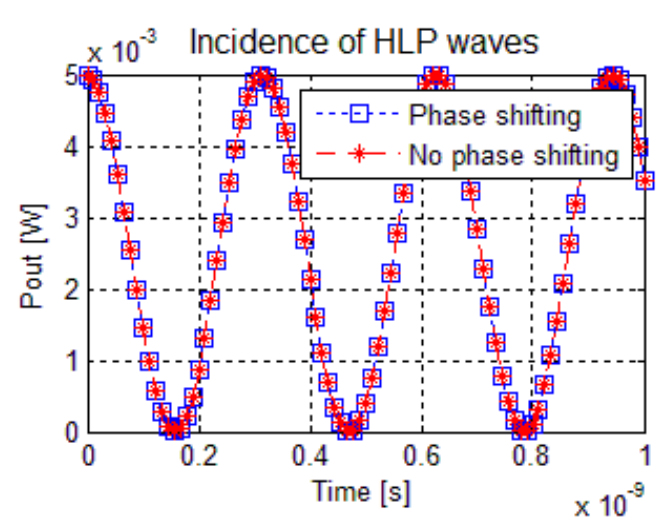

(a)

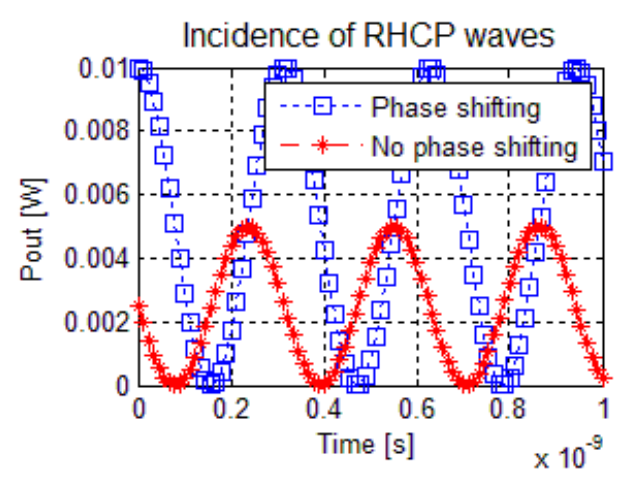

(c)

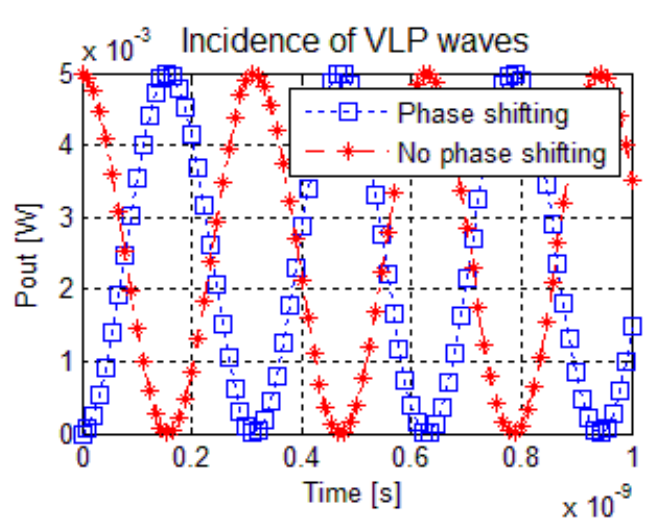

(b)

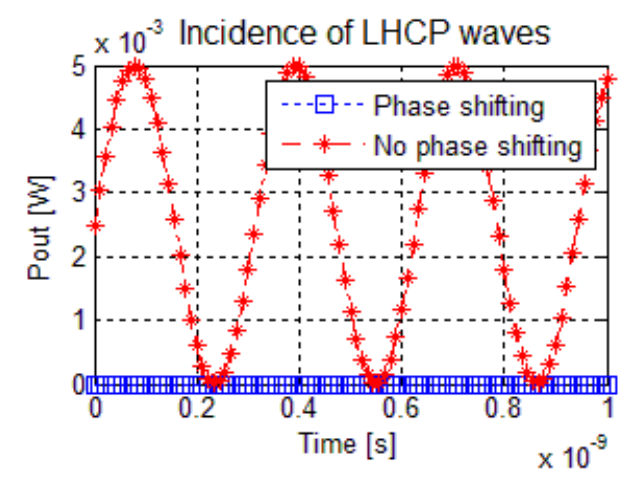

(d) 


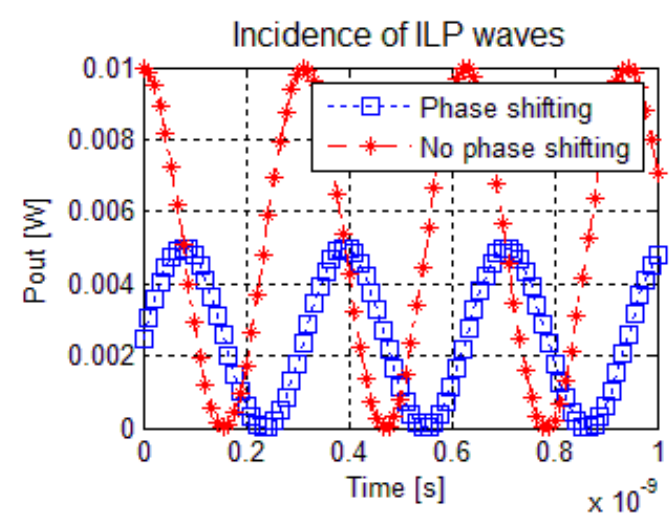

(e)

Fig. 8. Combiner output power on different incident waves occasions: (a) HLP, (b) VLP, (c) RHCP, (d) LHCP and (e) ILP.

\section{ANALYSIS}

The numerical calculation shows that for the case of HLP incident waves the polarization diversity set simply receives the signal through the $H$ antenna and transmits it through the combiner switch, without any attenuation, as in Fig. 8.a.

Fig. 8.b reveals that, for the configuration of delay of the signal generated on the $V$ antenna, there will be phase delay also on instantaneous electric power in case of VLP incident waves, which in no way would affect the operation of ESM systems.

In Fig. 8.c, for the case of RHCP waves, the power on the combiner switch output will double when there is delay on the signal generated in the $V$ antenna, since the two electrical components will have its peaks added at once. There will be no increased signal in the case without phase shifting.

If there is incidence of LHCP waves, there will be no signal intensification or attenuation when the configuration is without delay. However, for the example of combiner delaying the signal generated in $V$ antenna, the peak of an electric component would be added to the other's valley, which completely attenuate the signal at the output, as shown in Fig. 8.d. The set behavior on 8.c and 8.d shows that it synthetically simulates a RHCP antenna when there is phase delaying on the $V$ antenna. The delay on the $H$ antenna would simulate a LHCP antenna, instead of a RHCP one.

Fig. 8.e shows that when there is incidence of $45^{\circ}$ ILP signals on the configuration without delay, the components received by the two antennas are summed in phase, which causes the output power to be twice as high as it would be if the incident signal was VLP or HLP. An orthogonal ILP signal $\left(-45^{\circ}\right)$ would not be receivable, since its valleys and its peaks would cancel each other. Thus, an angular disposition of the array of $\pm 45^{\circ}$ with the ground would increase a VLP or HLP signal in $3 \mathrm{~dB}$.

This results show that the correct choice of the proper configuration of the combiner and of the angular disposition of the array would be advantageous in front of spirals' performance: (I) an angular disposition of $0^{\circ}$ of the array (one antenna on vertical and the other on horizontal), relative to the ground, with no phase shifting would receive VLP or HLP with the $9.5 \mathrm{dBi}$ from the mentioned modern bow-tie, and the mentioned modern spiral would receive them with $6.8 \mathrm{dBi}$ minus $3 \mathrm{~dB}$ 
equals to $3.8 \mathrm{dBi}$, which means a $5.7 \mathrm{~dB}$ better performance of the bow-tie array. The same configuration would receive both RHCP and LHCP with the same performance, which means $2.7 \mathrm{~dB}$ better performance than spirals (and remember that a single spiral would not be able to receive both of them); (II) an angular disposition of $0^{\circ}$ of the array with $90^{\circ}$ phase shifting on the signal generated on one antenna would receive VLP or HLP signals with no attenuation, as in statement (I), but a RHCP or LHCP signal would be received with $9.5 \mathrm{dBi}$ plus $3 \mathrm{~dB}$ equals to $12.5 \mathrm{dBi}$ by the array, again 5.7 $\mathrm{dB}$ higher than the spirals; (III) at last, an angular disposition of $\pm 45^{\circ}$ of the array, relative to the ground, with no phase shifting, would receive VLP or HLP with $9.5 \mathrm{dBi}$ plus $3 \mathrm{~dB}$ equals to $12.5 \mathrm{dBi}$, $8.7 \mathrm{~dB}$ higher than the $6.8 \mathrm{dBi}$ minus $3 \mathrm{~dB}$ equals to $3.8 \mathrm{dBi}$ performance that would be shown by a spiral. The disadvantage from statement (III) option is the fact that VLP or HLP signals must be chosen, while spirals receive both of them.

Electronic and mechanical maneuvers could be implemented to switch between the different angular dispositions and phase shifting configurations, in order to obtain the greatest possible advantages in front of different emitters.

This $8.7 \mathrm{~dB}$ higher intensity available power, applied on the ESM detection range equation [7], together with the simplest truncation effect issues on the array radiation patterns, provides the ESM system the capability of obtaining a more accurate measurement of DOA and other parameters from a range of 2.7 times the range than it would obtain with spirals, with less costs and time spent on design and production. The $5.7 \mathrm{~dB}$ factor found would multiply the range by 1.9 times and the $2.7 \mathrm{~dB}$ factor by 1.4 times.

\section{CONCLUSIONS}

The power level has crucial importance in a conflict between a radar and an ESM system. Although some polarization diversity devices applied to ESM systems have been develop in the 1980s, even today most of these military systems are conceived with spiral antennas.

This paper has shown that the spirals offer lots of inconveniences to those systems, like polarization matching issues, low gain, critical truncation effects for DOA measurement, high costs and time spent on designing, computer simulating and production.

In contrast, the absence of losses by polarization mismatching on a polarization diversity bow-tie antenna array was mathematically demonstrated. Its simpler truncation effect issues, favorable for DOA measurement, its faster and cheaper design, computer simulating and production were seen.

The results have shown that a polarization diversity bow-tie antenna array could provide for an ESM system a power $8.7 \mathrm{~dB}$ higher than a modern spiral would when confronting HLP or VLP radars, which would multiply by 2.7 the range of the system. They also presented that ESM systems equipped with the array would be able to confront both RHCP and LHCP radars, unlike those equipped with spirals. 
Taking into account the above mentioned factors, the replacement of traditional spiral antennas by polarization diversity antenna arrays would be convenient on the upcoming ESM system designs.

\section{ACKNOWLEDGEMENTS}

The authors would like to thank Adriane Marie Salm Coelho, Fernando Rangel de Sousa, Rodrigo Vinícius Mendonça Pereira and Daniel Basso Ferreira, who provided insight and expertise that greatly assisted the development of this work.

\section{REFERENCES}

[1] N. T. Duc, S. Shin. "A proposal antenna polarization diversity for LTE 2x2 MIMO in indoor testbed" in Proc. Seventh Int. Conf. on Ubiquitous and Future Networks, 2015, pp. 35-36.

[2] E. -S. Yang, H. -W. Son. "Dual-polarised metal-mountable UHF RFID tag antenna for polarisation diversity". Electronic Letters, vol. 52, issue 7, pp. 496-498, Apr. 2016.

[3] J. Zhu, S. Li, B. Feng, L. Deng, S. Yin. "Compact dual-polarized UWB quasi-self-complementary MIMO/diversity antenna with band-rejection capability”. IEEE Antennas and Wireless Propagation Letters, vol. 15, pp. 905-908, Sep. 2015.

[4] P. M. Ndao, Y. M. Erhel, D. Lemur, M. Oger, J. Le Masson. "First experiments of a HF MIMO system with polarization diversity" in Proc. Int. Conf. on Ionospheric Radio Systems and Techniques, 2012, pp. 1-5.

[5] K. Suzuki, K. Tanizawa, S. -H. Kim, S. Suda, G. Cong, K. Ikeda, S. Namiki, H. Kawashima. "Polarization-rotator-free polarization-diversity 4x4 Si-wire optical switch". IEEE Photonics Journel, vol. 8, issue 2, Feb. 2016.

[6] M. S. Khan, A. -D. Capobianco, A. Naqvi, B. Ijaz, S. Asif, B. D. Braaten. "Planar, compact ultra-wideband polarisation diversity antenna array". IET Microwaves, Antennas \& Propagation, vol. 9, issue 15, pp. 1761-1768, Dec. 2015.

[7] R. G. Wiley. ELINT: The Interception and Analysis of Radar Signals. Boston: Artech House Inc., 2006.

[8] A. A. Heidari, M. Simrooni, M. Nakhkash. "Analysis and Design of an X-Band Microstrip Patch Array Antenna for ESM Application", in Proc. Int. Conf. on Electromagnetics in Advanced Applications, 2007, pp. 559-562.

[9] V. Alleva, D. Baccello, M. Bartocci, B. Orobello. "Digital Antenna Unit for DA Analyis in ESM Systems" in Proc. $7^{\text {th }}$ European Radar Conference, 2010, pp. 479-482.

[10] Computer Simulation Technology. "Studio Suite". Internet: www.cst.com, 2016 [Apr. 10, 2016].

[11] V. H. Rumsey. Frequency independent antennas. New York: Academic Press Inc., 1966.

[12] H. Nakano, K. Kikkawa, N. Kondo, Y. Iitsuka, J. Yamauchi. "Low-profile equiangular spiral antenna backed by an EBG reflector". IEEE Transactions on Antennas and Propagation, vol. 57, issue 5, pp. 1309-1318, May 2009.

[13] C. A. Balanis. Antenna Theory: Analysis and Design. $3^{\text {rd }}$ edition. Hoboken: John Wiley \& Sons, Inc., 2005.

[14] H. Kogure, Y. Kogure e J. C. Rautio. Introduction to antenna analysis using EM simulators. Boston: Artech House Inc, 2011 .

[15] J. -Y. Zhao, Z. -Y. Zhang, N. -W. Liu, G. Fu, S. -X. Gong. "Wideband Unidirectional Bowtie Antenna with Pattern Improvement". Progress In Electromagnetics Research Letters, vol. 44, pp. 119-124, Jan. 2014.

[16] J. L. Volakis e K. Fujimoto. Small antennas: miniaturization techniques \& applications. New York: McGraw-Hill, 2010.

[17] S. Sitaraman, Y. Suzuki, C. White, V. Nair, T. Kamgaing, F. Juskey, S. Kim, P. Raj, V. Sundaram e R. Tummala. "Modeling, Design and Demonstration of Multi-Die Embedded WLAN RF Front-End Module with Ultra-miniaturized and High-performance Passives" in Proc. IEEE 64 $4^{\text {th }}$ Electronic Components and Technology Conference, 2014, pp. $1264-1271$.

[18] The Mathworks Inc. "Matlab R2010a”. Internet: www.mathworks.com/products/matlab, 2016 [Apr. 10, 2016 ]. 